\title{
UN ANÁLISIS DE LA ADAPTACIÓN AL CINE DE LA LISTA DE SCHINDLER POR PARTE DE STEVEN SPIELBERG
}

\section{AN ANALYSIS OF STEVEN SPIELBERG'S SCREEN ADAPTATION OF SCHINDLER'S LIST}

\author{
AUTOR \\ José Díaz-Cuesta \\ Profesor Universidad de La Rioja. Logroño (España). \\ jose.diaz-cuesta@unirioja.es
}

\section{RESUMEN}

Este artículo aborda el tema de la adaptación al cine por parte del director Steven Spielberg (1993) de la novela de Thomas Keneally Schindler's List, publicada originalmente con el título Schindler's Ark en 1982. Nos fijamos inicialmente en la breve crónica de Herbert Steinhouse, publicada en 1995, pero supuestamente escrita más de treinta años antes, que se centra en la figura de Oskar Schindler sin mencionar a Amon Goeth, y dando cuenta de cómo Schindler influyó en la muerte del Comandante local de la SS. Keneally no parte de ese relato, sino del que le transmiten de forma oral en un primer momento Leopold Page (antes conocido como Pfefferberg) y otros supervivientes a los que entrevista. Spielberg a su vez se basa inicialmente en la novela de Keneally, que completa con más relatos orales de testigos de los dramáticos sucesos que se narran. Aunque la película incluye algunos detalles ficticios -especialmente hacia el final del relato-, logra reproducir de manera fidedigna lo narrado por Keneally en su novela, cuya estructura principal coincide con la crónica de Steinhouse. 


\section{PALABRAS CLAVE}

La lista de Schindler - Estudio comparado - Cine y literatura - Thomas Keneally Steven Spielberg.

\section{ABSTRACT}

This journal article deals with the problem of Steven Spielberg's screen adaptation of Thomas Kenally's novel Schindler's List, originally published as Schindler's Ark in 1982. Firstly we focus on Herbert Steinhouse's journalistic account of the story, published in 1995, but supposedly written more than thirty years before. Steinhouse's account centres around Oskar Schindler, without mentioning Amon Goeth: he does tell how Schindler influenced on the killing of the local SS commander. Keneally's story is not based on Steinhouse's account, but on what is orally told to him firstly by Leopold Page (previously known as Pfefferberg) and then by other survivors he interviews. Spielberg departs from Keneally's novel, which is complemented by other oral accounts from witnesses of the dramatic events that are told. Although the film includes some fictitious elements -especially near its end-, it does manage to faithfully retell Keneally's novel, whose main structure coincides with Steinhouse's account.

\section{KEY WORDS}

Schindler's List - Comparative study - Literature and film - Thomas Keneally Steven Spielberg. 


\section{ÍNDICE}

1. Introducción

2. La crónica de Herbert Steinhouse

3. La novela de Thomas Keneally

4. El texto fílmico de Steven Spielberg

5. Conclusiones

6. Referencias

\section{Introducción}

La historia de Oskar Schindler, en las diversas formas en que nos ha llegado, resulta de especial interés para el público iberoamericano, en especial el argentino, ya que durante varios años posteriores a la Segunda Guerra Mundial Schindler vivió en ese país, en el que su viuda, Emilie Schindler, permaneció hasta unos meses antes de su propia muerte, acaecida en 2001: Oskar había abandonado el país y a su mujer anteriormente, en 1957 (Brecher, 1994, p. XXXVII). Schindler y su esposa compartieron destino iberoamericano con muchos otros nazis, pero en este caso se trataba de nazis que durante la guerra evolucionaron en su actitud hacia el pueblo judío hasta el extremo de poner en peligro su fortuna y su vida para proteger a varios centenares de judíos.

En este artículo no nos centramos en la vida de Oskar Schindler tras la guerra, sino en lo sucedido durante la misma, según ha sido transmitido por Herbert Steinhouse (1995), Thomas Keneally (1982) y Steven Spielberg (1993). Tomando la crónica de Steinhouse como punto de partida, nos interesa analizar cómo se aproxima cada autor a la figura de Oskar Schindler y a los eventos que la rodearon durante la Segunda Guerra Mundial. 
Para ello nos fijamos inicialmente en la crónica de Steinhouse, a continuación nos detenemos en la novela de Keneally, y por último nos centramos en la película de Spielberg, prestando especial atención a la diferenciación que se produce entre hechos reales y ficticios en los relatos de los dos últimos autores.

\section{La crónica de Herbert Steinhouse}

El relato periodístico que Herbert Steinhouse publica en 1995 había sido escrito más de treinta años antes, después de conocer al propio Schindler y a su esposa, y de corroborar la historia con supervivientes de la lista y con actas notariales (Fensch, 1995a, p. 13).

Quien se haya acercado a las versiones de Keneally y de Spielberg notará una omisión importante en el relato de Steinhouse: en el mismo no se menciona a Amon Goeth. La explicación puede hallarse en el hecho de que el relato es breve y conciso, y tiene el afán periodístico de centrarse en la figura de Oskar Schindler y en sus acciones durante la Segunda Guerra Mundial. Cuando Steinhouse entrevistó al matrimonio Schindler y corroboró su historia con documentos legales y testimonios de personas salvadas del exterminio por Oskar Schindler, no necesitaba incluir la figura de un villano porque los hechos estaban muy recientes y no hacía falta personificar el mal de los nazis con un ejemplo como el de Goeth, aunque el comandante ciertamente existió y era objeto de las peores pesadillas de los supervivientes a su campo de concentración.

El resto del relato coincide en lo fundamental con lo narrado por Keneally y por Spielberg, con la diferencia de que en la crónica de Steinhouse sí se menciona el hecho de que Schindler "had decided that they would have to get rid of the local SS commander" (Steinhouse, 1995, p. 33). Schindler no mató a ese otro comandante con sus propias manos, y quienes lo hicieron actuaban de forma preventiva en defensa propia y de los Schindler, en su verdadera huida, no en la que dramatiza 
Spielberg al final de su película. Pero contribuyó a que se produjera un asesinato, algo de lo que no dan cuenta ni Keneally ni Spielberg.

\section{La novela de Thomas Keneally}

La novela presenta el problema inicial de su catalogación: ¿debemos considerarla una obra de ficción o una serie de hechos dramatizados? En inglés se suele utilizar el término faction para referirse a este tipo de combinaciones de hechos reales y ficticios, fact and fiction. Fritz Lanham cita al autor de la novela refiriéndose a este asunto:

\footnotetext{
"Keneally calls it a "documentary novel," "a nonfiction novel," a "faction," and professes to be "astounded that people are so mystified about where it fits, given that you have had a number of famous American books that are in this genre, like In Cold Blood and The Right Stuff and The Executioner's Song and The Armies of the Night."' (Lanham, 1995, p. 43)
}

El término novela que hemos escogido para titular este apartado puede seguir siéndonos útil, ya que existen, como afirma Keneally, novelas de no-ficción (nonfiction novels en inglés). Aunque, por otro lado, a la novela se le concedió el premio Pulitzer de 1982 como obra de ficción. Lanham se fija en lo que dice el propio libro:

"The cover of Schindler's List calls it a novel-a label that has occasioned considerable confusion. Actually, it's a carefully researched, true account that incorporates some of the storytelling of fiction." (Lanham, 1995, p. 43)

Y nos recuerda el método seguido por Keneally para documentarse: 
"What dialogue there is in the book is based on survivors" recollections of what was said, or what they were told was said. Keneally did not fuse events for dramatic purposes. Indeed he sent parts or all of his manuscript to some 20 Schindler survivors to make sure he was telling the story the way it happened." (Lanham, 1995, p. 43)

Keneally, por tanto, coincide en gran medida con la manera en la que se informó Steinhouse, con la salvedad de que este último pudo entrevistar a Oskar Schindler, algo que le resultó imposible al autor australiano porque Schindler había fallecido en 1974 (Lanham, 1995, p. 42). La etiqueta que le podamos poner a la novela no es de extrema importancia, dado que se nos certifica que está basada en hechos reales. Es más interesante estudiar la manera en que esos hechos son incluidos en la misma. Para ello nos vamos a detener por un momento en su autor, y a continuación procedemos a analizar las herramientas principales de las que hace uso para conservar la distancia entre realidad y ficción.

Como acabamos de mencionar, Thomas Keneally es australiano. Estuvo preparándose para convertirse en sacerdote católico, pero finalmente no tomó los hábitos. Sus inclinaciones políticas se decantan por el Australian Republican Movement, cuyo propio nombre indica que se trata de una agrupación republicana. De hecho Keneally ha publicado varios escritos en defensa de una república independiente del Reino Unido (Ponce, 1995, pp. 38-40). El que sea católico y republicano es relevante en relación con el tema que nos ocupa. Por un lado, el ser católico le distancia en cierta medida del judaísmo, o al menos le sitúa en una posición menos comprometida que la de los supervivientes o el propio Spielberg, que también es judío: pero coincidiría en su inclinación religiosa con la de Schindler, de manera que la distancia se acorta en relación al protagonista del relato. Por otro lado, el hecho de que desee un estado republicano en Australia le colocaría al lado 
de los judíos, que también han reclamado -y han conseguido- un país propio e independiente de cualquier otra potencia ${ }^{1}$.

Volviendo a la novela propiamente dicha, su estructura se basa en 39 capítulos, una nota del autor, un prólogo y un epílogo. La historia está contada de manera lineal, excepto por el prólogo, como veremos cuando analicemos los comienzos de libro y película. Hay pocos flashbacks en el resto del relato. La idea general de contar la historia de un grupo de gente que es salvado está emparentada con una tónica general del Antiguo y del Nuevo Testamento, en especial del primero, en el que se forman grupos o pueblos desde el comienzo de los tiempos. La relación con la Biblia se ve reforzada por el hecho de que Keneally, en su prólogo, define el libro como "the pragmatic story of the triumph of good over evil" (p. 15). La novela está dedicada "To the memory of Oskar Schindler, and to Leopold Pfefferberg who by zeal and persistence caused this book to be written" (p. 7).

Podemos considerar que el libro consta de tres comienzos diferentes, a los que acabamos de aludir. El primero de ellos sería la Author's Note, en la que Keneally detalla la manera en la que le fue dada a conocer la historia de Schindler y se justifica por usar un estilo de novela documental. A continuación viene el Prologue, que se localiza en una fiesta en la villa de Goeth en el otoño de 1943. La historia comienza, por tanto, in media res. A ese salto en el tiempo le sigue el primer capítulo, que repasa la infancia tanto de Goeth como de Schindler, hasta el momento en el que este último se convierte en colaborador del partido nazi.

A partir de ese primer capítulo, Goeth será comparado sistemática y repetitivamente con Schindler. En el capítulo 20, por ejemplo, podemos leer que "Amon was Oskar's dark brother" (p. 188).

\footnotetext{
${ }^{1}$ No entraremos aquí en la relación que Israel tiene con los Estados Unidos de América: sería el tema de otros artículos.
} 
Cada uno de los capítulos siguientes aparentemente se basa en relatos de varios testigos, incluyendo cincuenta supervivientes de la lista, desde cuyo punto de vista se focaliza la historia. Sus testimonios levantan un edificio de diferentes voces que contribuye a la veracidad de la historia.

Por último, cabe citar que tal vez el recurso más destacable que utiliza Keneally para diferenciar entre hechos reales y ficticios es la inclusión de expresiones para por ejemplo especificar lo que ha sido "documented" (p. 43), lo que "is most likely" (p. 21), y lo que es parte de la "legend" (p. 376). Podemos concluir, por tanto, que en la novela de Thomas Keneally es posible distinguir entre lo que fue real y lo que es meramente ficción.

\section{El texto fílmico de Steven Spielberg}

El concepto de autoría ha sido interpretado múltiplemente según la base teórica con la que se considere ${ }^{2}$. Incluso sin llegar a ese tipo de consideraciones, hablar de un autor cuando nos referimos a una película puede llevar a equívocos, según nos fijemos en las diversas aportaciones individuales de las que consta cualquier texto fílmico. Cuando encabezamos este apartado como "El texto fílmico de Steven Spielberg" nos estamos refiriendo a la persona responsable del resultado final de la película, y de coordinar, equilibrar $y$, desde luego dirigir el trabajo en este caso del guionista Steven Zaillian y del director de fotografía Janusz Kaminski, además de guiar la interpretación de los actores y actrices que dan vida a los personajes, por mencionar sólo algunos de los aspectos que Spielberg ha tenido que controlar para realizar esta película.

\footnotetext{
2 Véase BRANIGAN, Edward R. (1992): Narrative Comprehension and Film. Routledge. Londres y Nueva York. Pág. 86-124.
} 
El texto fílmico es, pues, de Steven Spielberg: un Steven Spielberg que a raíz de la realización de esta película redescubre sus orígenes como niño judío ${ }^{3}$. El director no escoge, no obstante, un relato en el que los protagonistas principales son los judíos, sino el que nos ocupa, en el que el personaje más importante es lo que se solía denominar como un alemán bueno. Esta aparente contradicción no nos debería desviar del otro texto que subyace tras la historia de Oskar Schindler, que es el Holocausto: ahí de nuevo los orígenes judíos de Spielberg han tenido que influir emocionalmente en muchas de las decisiones que haya tomado.

Parece que el destino de esta historia era convertirse en una película de Hollywood. Anne Thompson narra el camino inicial del relato tratando de adoptar forma de guión:

"Page [Leopold Pfefferberg] had first helped sell Schindler's story to MGM in the 1960s. The studio hired Casablanca coscreenwriter Howard Koch to work on the film, but the dubious character of Oskar Schindler-a womanizing, boozing bear of a man who profited from the war by employing Jews-may have made his story a difficult sell. The project was dropped and lay dormant for years before Spielberg decided to take it on." (Thompson, 1995, p. 67)

Spielberg adquirió los derechos de la película poco después de que se publicara la novela, pero tardó años en decidirse a afrontar el reto de filmarla:

"Page, 80, insists that from their first meeting, Spielberg knew he would do the movie. "When I met him," he recalls in thickly accented English, "I asked him, 'Please, when are you starting?' He said, Ten

\footnotetext{
${ }^{3}$ Es posible hallar múltiples ejemplos de esta vuelta a los principios de Spielberg en las diversas entrevistas y citas del director que aparecen en FENSCH, Thomas (ed.) (1995b): Oskar Schindler and His List: The Man, the Book, the Film, the Holocaust and Its Survivors. Paul S. Eriksson. Forest Dale. Vermont.
} 
years from now." But as those years passed, Page worried that he wouldn't live to see the film.

"Schindler's was on my guilty conscience," says Spielberg. "Page was heaping on the fact that he was going to die."' (Thompson, 1995, p. 67)

La película es fiel a la novela en la manera en la que se aproxima a las historias que se van contando: hay un relato principal, que tiene como protagonista a Schindler (Liam Neeson), y poco a poco vamos descubriendo lo que está sucediendo alrededor suyo. En numerosas ocasiones sentimos que la cámara se desvía del argumento y adopta un tono documental, recorriendo hileras de gente o adentrándose en zonas que escapan a la visión de los judíos o del propio Schindler, como cuando se nos muestra lo que se hace con los contenidos de las maletas que los presos han tenido que abandonar (F01).

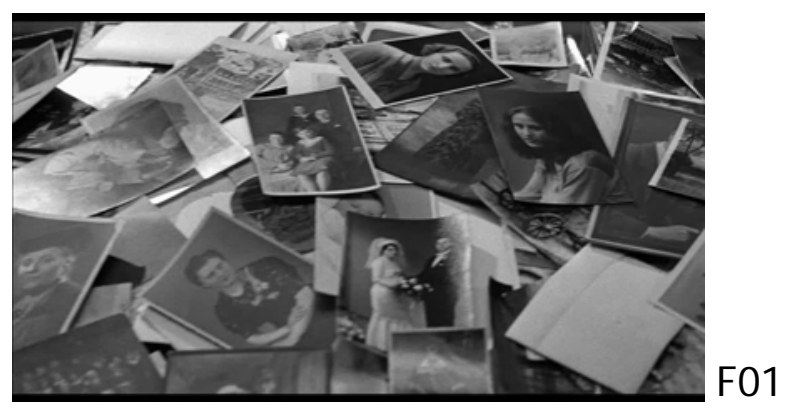

El uso del blanco y negro ahonda en el carácter simuladamente documental de algunos pasajes del film, o al menos trata de reproducir el tipo de película que se podía realizar en la época que se retrata. El blanco y negro destaca aún más porque contrasta con algunos momentos en los que sí se ven elementos en color en el film. Por un lado está la secuencia inicial, a la que nos referimos más adelante, y la final, en la que actores y actrices acompañan a las personas reales a las que representan en el relato. No obstante, el momento en color al que más se suele aludir es al que retrata únicamente en color, con un abrigo rojo, a una niña a la que sigue la mirada 
de Schindler desde su punto de observación durante la liquidación del gueto. La cámara no sólo nos muestra lo que ve Schindler (F02), sino que se adentra en una casa (F03), de manera que el espectador llega a estar más próximo a la niña visualmente de lo que lo pudo estar el propio Schindler, en un intento de alcanzar el grado de empatía que en ese momento podía sentir el empresario.
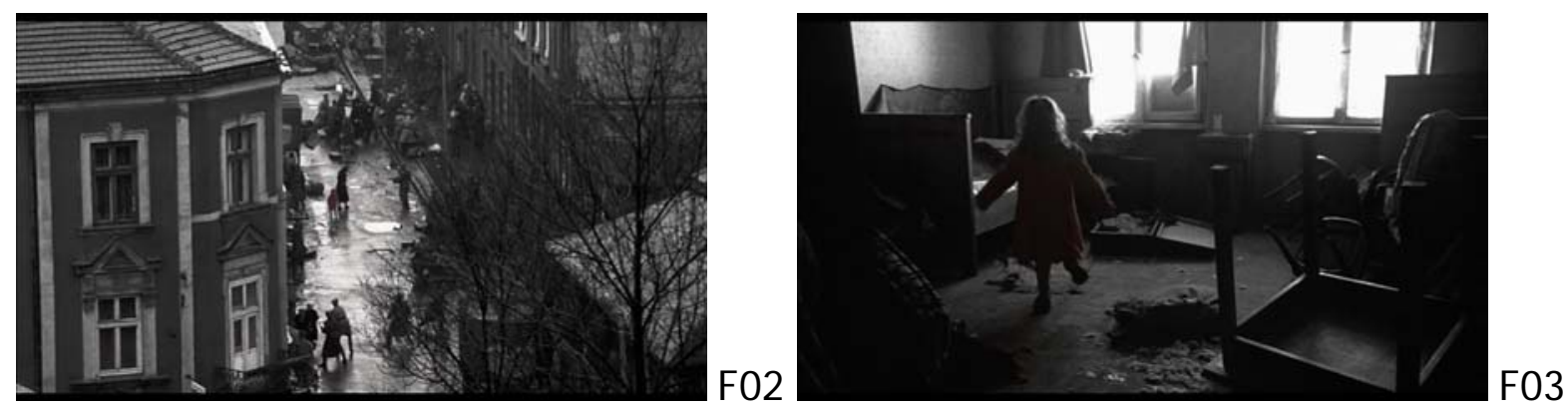

La niña volverá a aparecer, ya muerta, transportada en una carretilla, pero manteniendo el tinte rojizo de su vestimenta (F04).

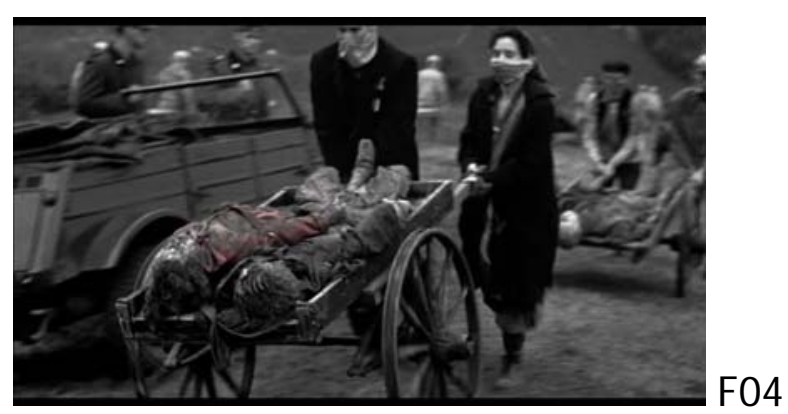

Este uso selectivo del color no es nuevo en la generación de cineastas en la que podríamos incluir a Spielberg, pues ya había sido utilizado con agudeza por el director Francis Ford Coppola para retratar los peces de Rumble Fish (Coppola, 1983). Sin llegar a la sutileza de mezclar color y blanco y negro, el propio Spielberg ya había seguido a un coche rojo en Duel (1972) (F05), a un corazón alienígena rojo en E.T. (1982) (F06) y a un niño vestido de rojo entre la multitud en Empire of the Sun (1987) (F07). 

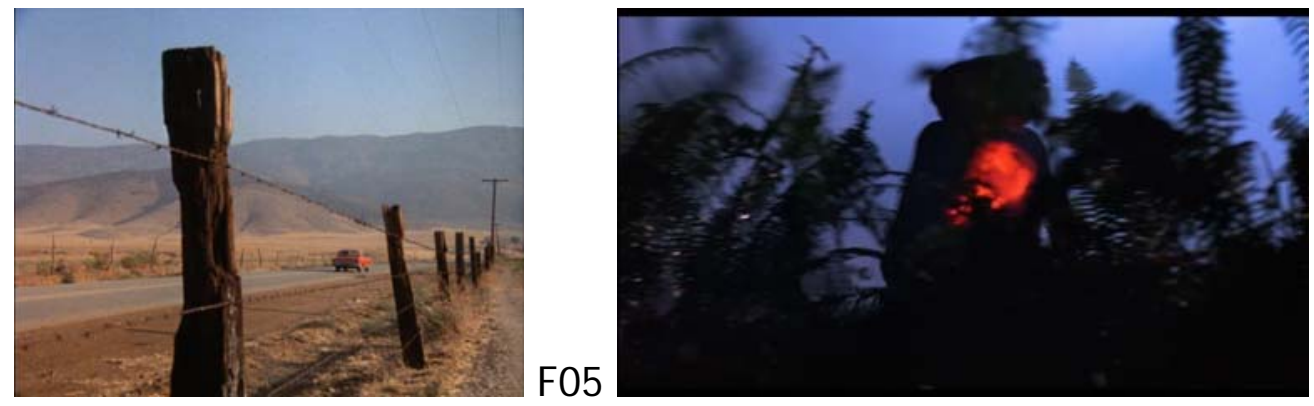

F06

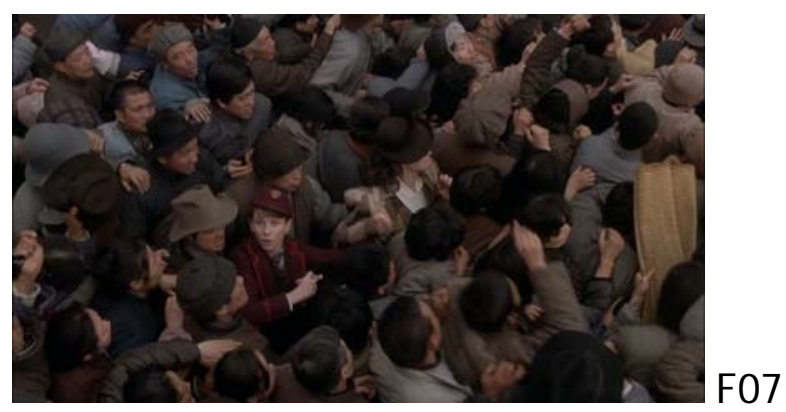

El film también destaca por numerosos fragmentos rodados cámara en mano, de nuevo intentando reflejar el espíritu de inmediatez del documental o del reportaje. La película se vuelve más clásica en el uso del montaje en paralelo, normalmente utilizado para contrastar dos escenas que se asemejan pero que guardan grandes diferencias, como cuando Schindler se establece en la casa de una familia judía (F08), a la que vemos cómo se le asigna un espacio ínfimo (F09), o cuando Schindler permanece en un club (F10) y mientras tanto se está casando una pareja judía $(\mathrm{F} 11)^{4}$.

\footnotetext{
${ }^{4}$ En realidad aquí se produce un montaje a tres bandas, ya que también se incorpora el interrogatorio retórico que Goeth le hace a Helen Hirsch (Embeth Davidtz), escena a la que nos referimos a continuación.
} 
Díaz- Cuesta, José (2003): Un análisis de la adaptación al cine de La Lista de Schindler por parte de Steven Spielberg. $\mathbf{N}^{\circ} \mathbf{1 0}$. Noviembre. Año VII. Páginas: $30-52$

ISSN: 1576-3420 DOI: http://dx.doi.org/10.15198/seeci.2003.10.30-52
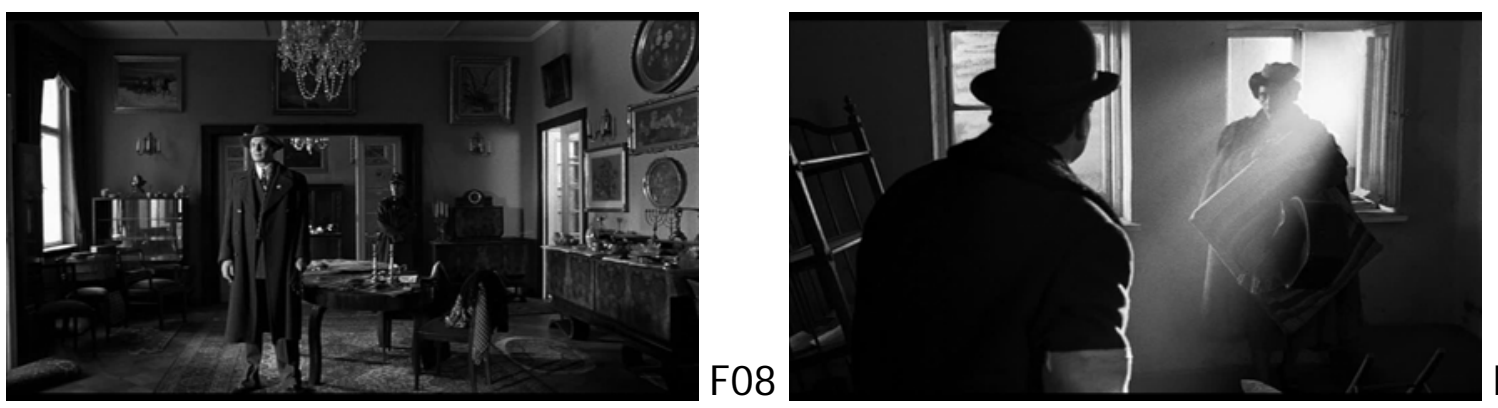

F09
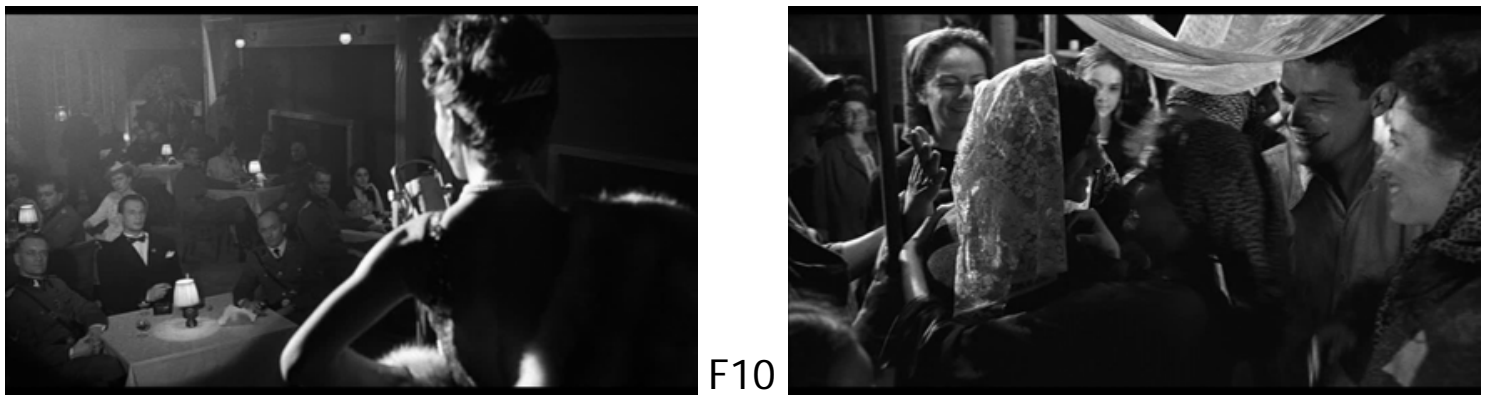

F11

El paralelismo también puede localizarse entre escenas separadas por el metraje de la película, como las dos entrevistas o interrogatorios a los que es sometida Helen Hirsch por Schindler (F12) y por Goeth (Ralph Fiennes) (F13).
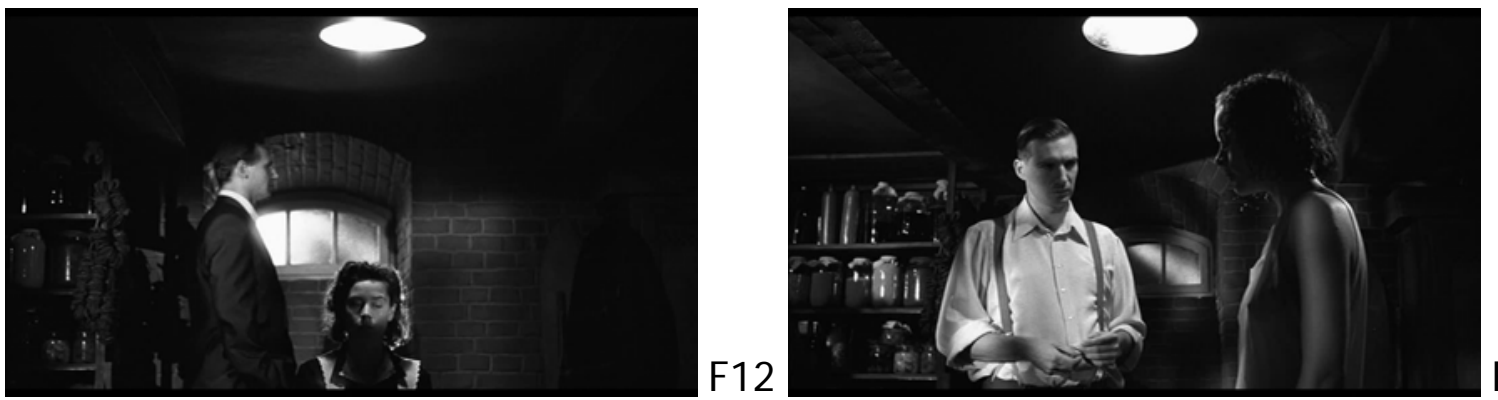

F13

El paralelismo llega a estar, ya no en el montaje, sino en la composición del propio plano, como esa imagen casi simétrica, como de un espejo, en la que Goeth y Schindler están sentados hablando, regateando sobre el precio de unos seres humanos (F14). 


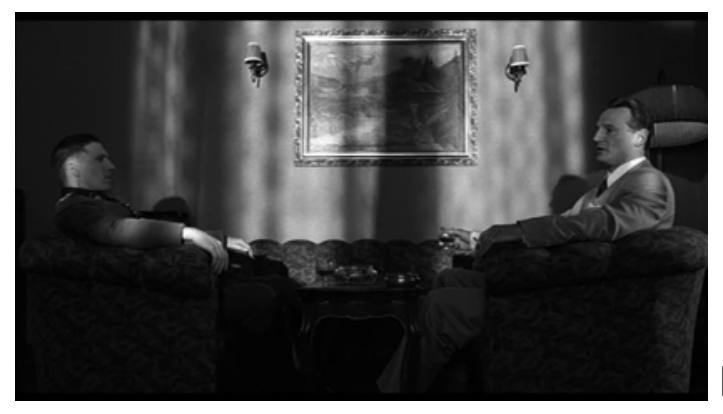

F14

Antes de analizar la manera en la que comienza el film, nos vamos a centrar en un elemento que alguien podría considerar como extra-fílmico, pero que es lo que más probablemente veamos antes que la propia película cuando accedemos al cine. Nos estamos refiriendo al cartel de la película (F15).

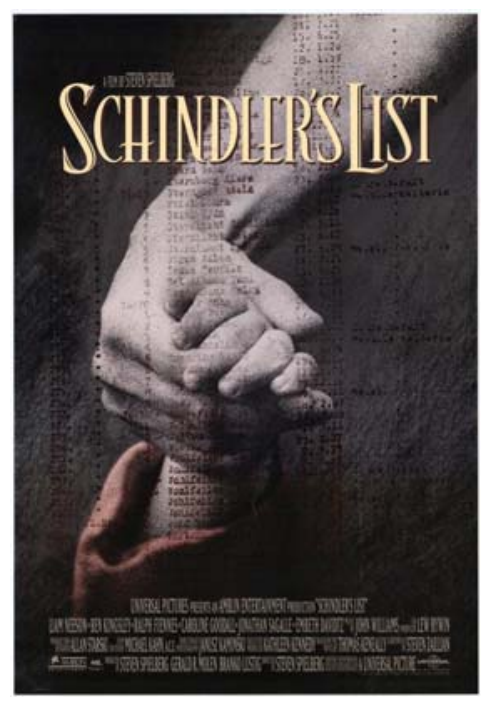

F15

La imagen general es la de dos brazos cuyas manos están entrelazadas. La mano de la parte superior es grande y aparece desnuda, mientras que la de abajo aparece cubierta parcialmente por una manga rojiza que contrasta con el resto de la imagen, que aparece en tonos sepias. El brazo superior recibe la mayor parte de la iluminación, mientras que el pequeño, junto con su mano, aparece difuminándose en la oscuridad de la parte inferior del cartel. Logramos ver todo esto a través de una lista de nombres y cifras que se asemeja mucho a la lista a la que alude el título de la película, y que en la misma podremos ver en manos de Schindler y de Itzhak Stern 
(Ben Kingsley). El cartel está invitando al espectador a mirar más allá de la lista, en donde lo inimaginable puede ocurrir. Es discutible si el brazo superior pertenece a Schindler o no, pero lo que arroja pocas dudas es que el inferior pertenece a la niña de rojo, cuya figura es resaltada no sólo en el film, sino también en la novela de partida. Sin embargo, el cartel presenta algo que físicamente no llega a suceder en la película: en ningún momento se observa a mano alguna sujetando la de la niña, que fallece finalmente. El cartel nos muestra a un adulto sujetando una de las millones de manos que no sobrevivieron al Holocausto, una mano que procede de lo insoportable, de lo que no resiste a la observación, del horror y de la muerte. En la película no se alude explícitamente a todos esos judíos muertos hasta el final de la misma, en el que se recuerda que murieron seis millones de personas.

El título de la película es un recurso por sí mismo, ya que el film, aún más que la novela, está plagado de escenas y planos en las que podemos ver filas, listas de gente, bien en persona o sobre el papel. Todas ellas se oponen a una sola fila, a la única lista que significa vida: la de Schindler. Louis Giannetti relaciona estas listas con el uso de grandes angulares por parte de Spielberg:

"Wide-angle lenses are used whenever deep-focus photography is called for. Objects a few feet from the lens as well as those in the "depth" of the background are in equal focus, reinforcing the interconnectedness of the visual planes. Deep-focus photography tends to be objective, matter-of-fact, unromantic. The foreground is not necessarily more important than the mid- or background. [...] Because deep focus allows for the repetition of visual motifs into infinity, Spielberg is able to suggest that Jews all over Europe were being herded in a similar manner, but their fate was not so lucky as Schindler's Jews." (Giannetti, 1996, p. 29) 
En cuanto a la manera en que comienza la película, podemos afirmar que el inicio del film resulta más misterioso que el de la novela, aunque no deja de ser convencional. Como sucede con el libro, podemos referirnos a tres comienzos consecutivos. La primera secuencia es en color, y de esta manera se relaciona de manera circular con la última, en la que aparece la tumba de Schindler. Ambas escenas funcionan como marco parentético para el resto de la película. Pero en primer lugar no vemos nada, sólo esa oscuridad que nos remite a la parte inferior del cartel y al momento sin iluminación de la escena de la ducha: elocuente oscuridad que habla por sí misma, por todas esas otras ocasiones en las que de aquellos aspersores salió gas en lugar de agua. A la oscuridad le sigue la luz en forma de cerilla, sujeta por una mano anónima (F16a), que transmite esa luz a sendas velas (F16b).
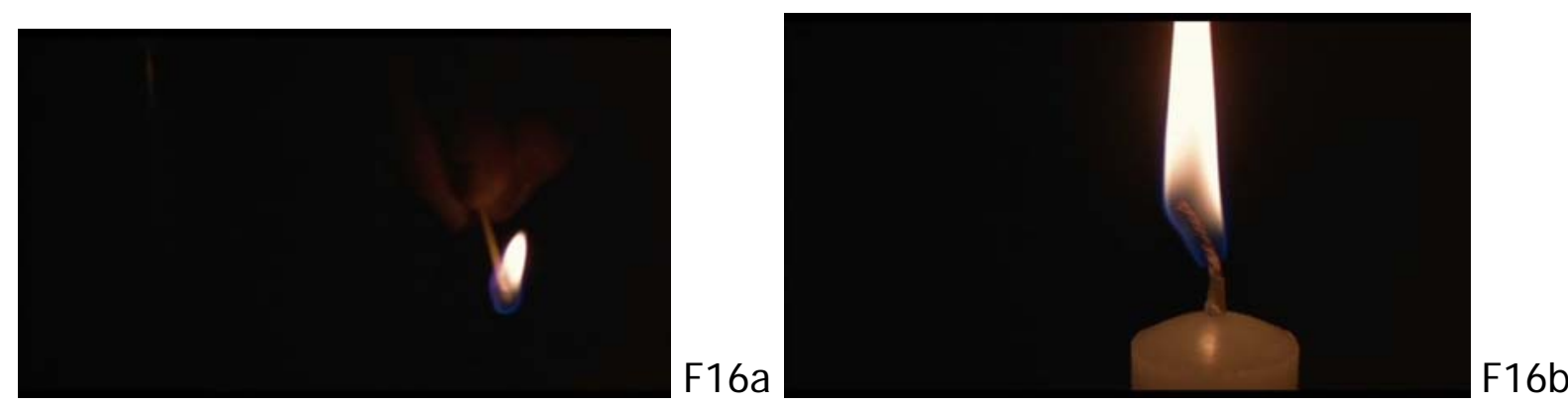

Al final de la secuencia, después de que hayamos podido ver a varias personas judías celebrando el Sabbath (F17), sólo queda una luz que se apaga suavemente, su humo convertido ya en blanco y negro (F18), más negro que blanco, como el humo del tren del plano siguiente (F19).

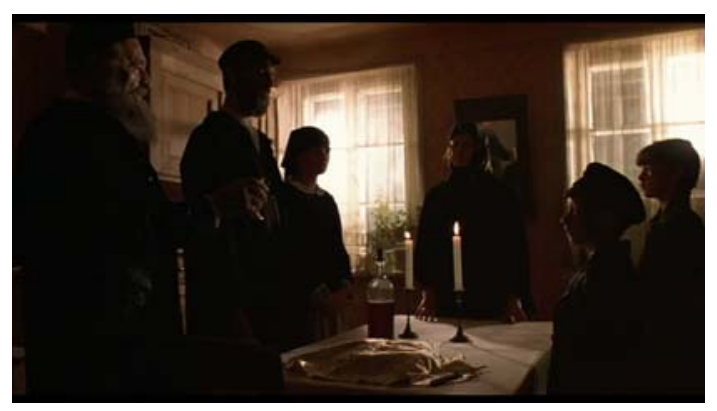



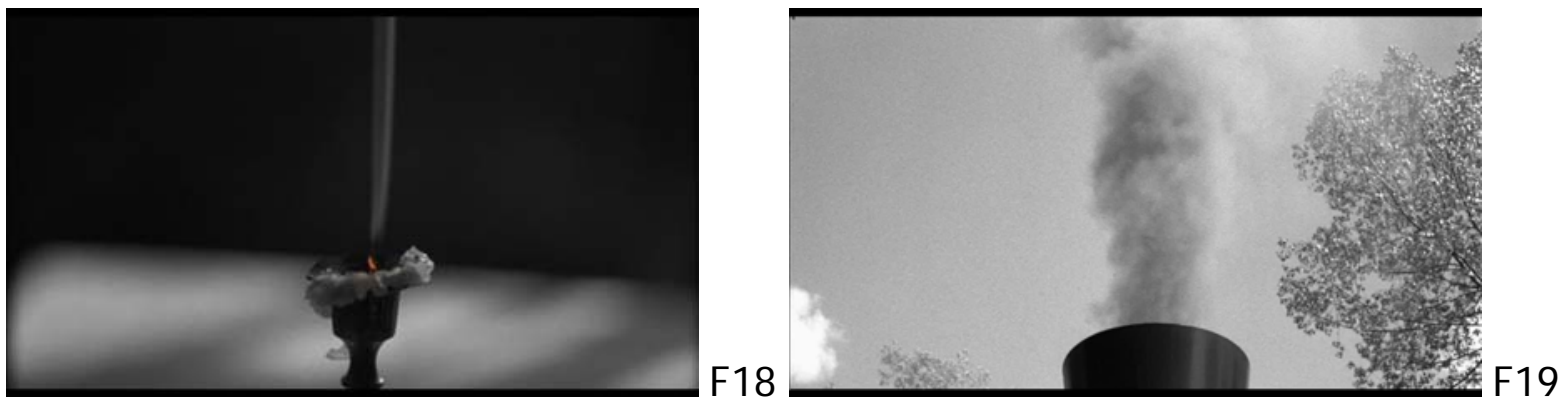

Ambas escenas, la de las velas y la de la estación, anuncian prolépticamente el humo que veremos salir de las chimeneas de los crematorios donde se incineraba a los judíos. Pero de momento sólo se nos muestran los primeros ejemplos de listas, llenos de nombres (F20) y de caras (F21).
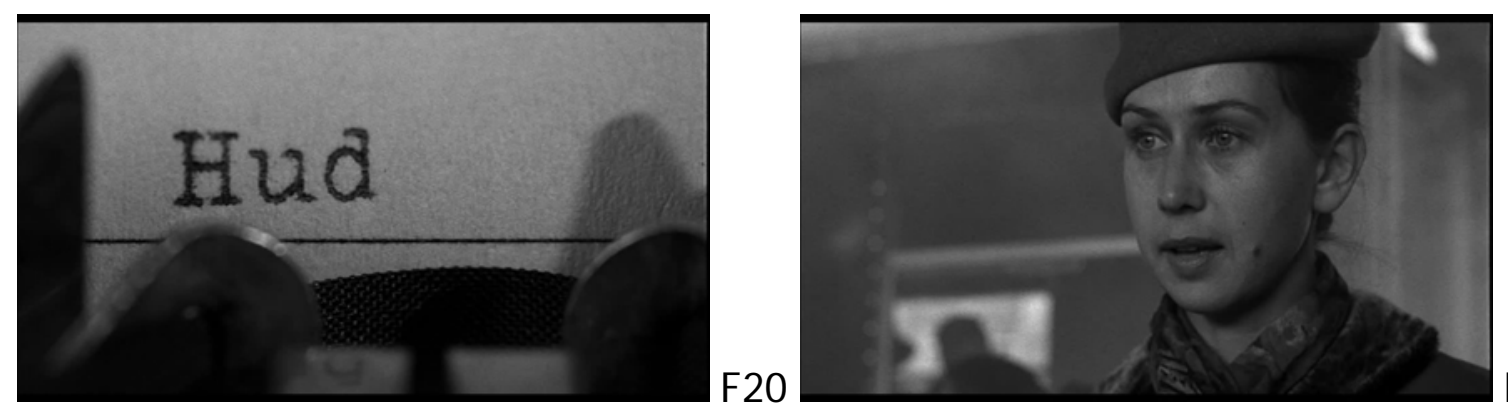

F21

Esas listas se contraponen a la misteriosa manera en la que se introduce al personaje de Schindler en las dos escenas siguientes, en lo que sería nuestro tercer comienzo. En la primera de ellas observamos detalles de alguien que puede elegir entre varias joyas y ropas caras para vestirse (F22). El broche de la solapa nos revela que se trata de un nazi (F23).
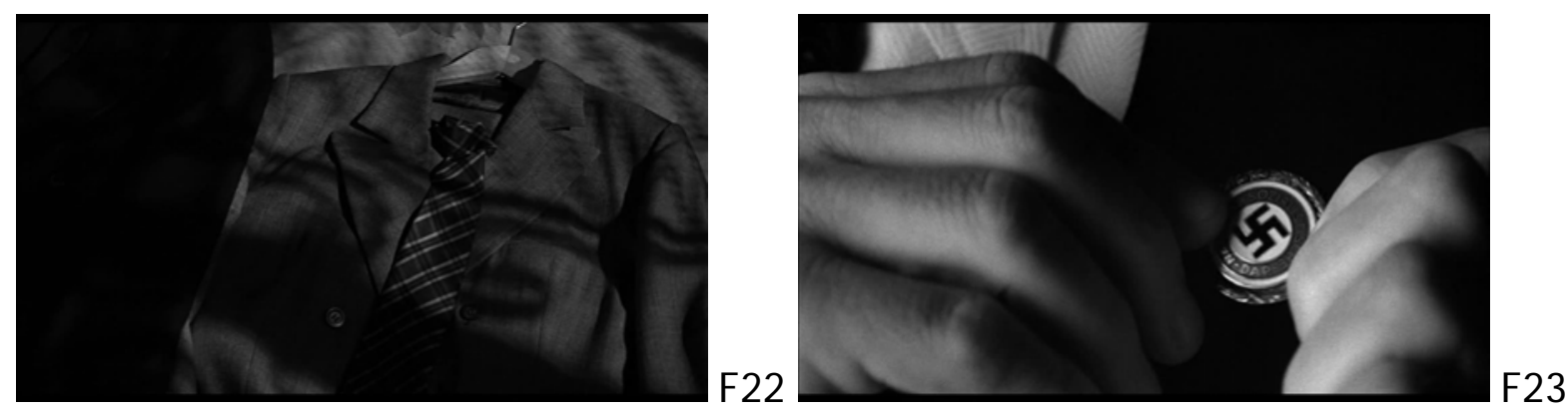
Su cara y su nombre, empero, no serán descubiertos hasta que nos encontremos bien avanzados en la siguiente escena. De hecho, nadie le conoce en el club al que acude. Llegamos a ver su cara (F24) y averiguar su apellido: Schindler. Entre ambos momentos hay un plano en el que le vemos desde el punto de vista del maitre, que está preguntándoles a sus camareros si conocen al recién llegado (F25). Ese plano muestra a Schindler a través de la cruz que forman las enmarcaciones de los vidrios de la puerta: un símbolo, el de la cruz, que le remite a su origen católico.
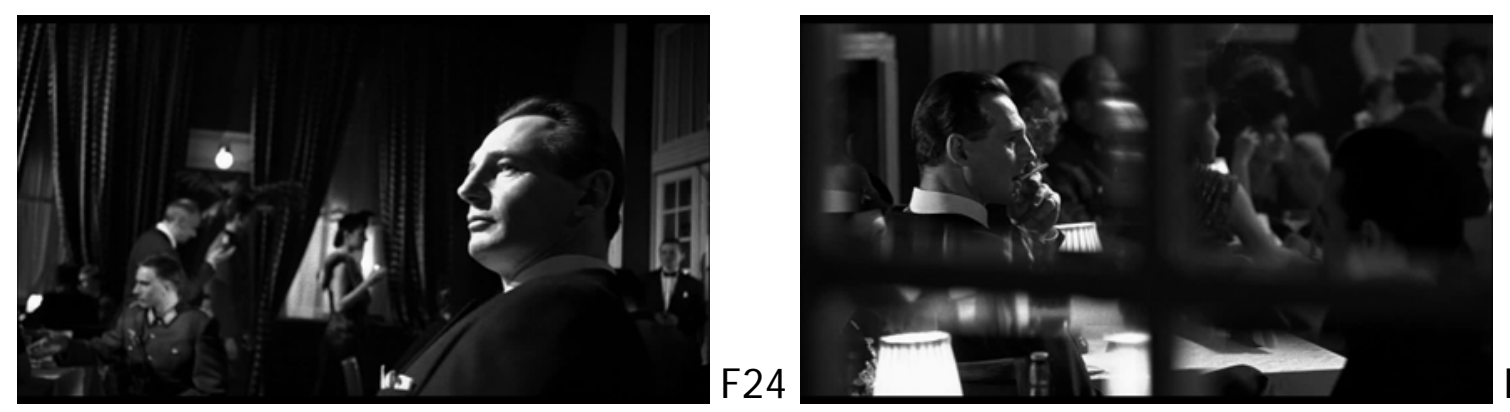

F25

Si relacionamos este triple comienzo con el también triple de la novela que hemos analizado en el apartado anterior, podemos concluir que, aunque cambie la forma en la que se narra, se cuenta una historia similar, el novelista dando cuenta de los hechos desde el principio, el cineasta creando un misterio.

Como es lógico, en la novela es posible encontrar muchos más detalles que en el film. Entre esos detalles se encuentra la relación que se establece entre Schindler y el movimiento de la Resistencia, relación que en la novela logra mostrar a Schindler no sólo como un salvador individual sino también como parte de un grupo. En la novela también se relatan crueles torturas que Spielberg no se atreve a mostrar, como las ejemplificadas por perros comiendo a personas (p. 204) o por los latigazos que son obligados a darse entre sí los pertenecientes a nacionalidades diferentes ( $p$. 280). 
En cuanto a las adiciones del film, sólo hay una digna de destacar, que ha sido criticada por Keneally. Nos referimos a la escena, al final de la película, en la que Schindler clama por las pocas personas más que podría haber salvado. Pero Spielberg tenía sus razones para incluirla:

"It was absolutely necessary," insists Spielberg. He's not speaking for himself, he's speaking for all of us, what we might do someday." (Thompson, 1995, p. 73)

Coincidimos con el director, y respaldamos su elección porque de esa manera consigue un efecto catártico, que es una de las convecciones del cine y del teatro.

Volviendo a la fidelidad a los hechos narrados, la intención del director refuerza lo que hemos apuntado sobre la veracidad de la película. En palabras de Spielberg:

"I came to realize, the reason I came to make the movie, is that I have never in my life told the truth in a movie. [...] That was one of the things I thought: if I'm going to tell the truth for the first time, it should be about this subject." (Ansen, 1995, p. 64)

\section{Conclusiones}

Podríamos haber continuado con el mismo procedimiento de comparar novela y película, con análisis textuales pormenorizados de momentos similares en ambas obras, pero eso supondría alargarnos más allá de los límites de espacio de un artículo. No obstante, tras la revisión general a la que hemos sometido a los dos textos, podemos concluir que tanto novela como película comparten el mismo espíritu (Seger, 1993, p. 30). 
En cuanto a la relación que ambas obras guardan con la crónica periodística de Steinhouse, las mayores diferencias estriban en que en este relato anterior se omite la figura de Amon Goeth y en cambio se cita un asesinato de un oficial nazi, asesinato al que contribuyó Oskar Schindler sin llegar a ser la mano ejecutora. Podemos concluir que crónica y película guardan una mayor similitud, ya que las dos están focalizadas en el personaje de Schindler, mientras que la novela está más polarizada, ya que en muchos momentos se ofrece tanto la visión de Schindler como la de Goeth.

El recorrido final de cualquier obra se realiza entre la misma y quien la recibe. En el caso del público iberoamericano, como en la mayor parte del mundo ${ }^{5}$, la historia que se narra en crónica, novela y película ha sido seguida ya por varias generaciones de lectores y espectadores, en un movimiento que va desde los padres hacia los hijos. Ciertamente, la película es el texto que más audiencia ha tenido, no sólo a través de su proyección en el cine, sino también gracias a las ediciones en vídeo y en DVD de la misma, así como en sus retransmisiones televisivas. Lo que comenzó como un misterioso gesto altruista por parte de Schindler se ha convertido en un símbolo de la bondad del ser humano, incluso cuando a su alrededor las personas dejan de tener esa consideración y se convierten en víctimas o verdugos. No queda sino esperar que seamos capaces de impedir que algo semejante vuelva a suceder.

\section{Referencias}

ANSEN, David (1995): "Spielberg's Obsession" en FENSCH, Thomas (ed.): Oskar Schindler and His List: The Man, the Book, the Film, the Holocaust and Its Survivors (pp. 56-64). Paul S. Eriksson. Forest Dale, Vermont. [Publicado originalmente en (1995): en Revista Newsweek, 20 de diciembre].

\footnotetext{
5 Para comparar la recepción inicial de la película en Alemania, Israel y Francia véase, respectivamente, Weissberg (1997), Bresheeth (1997) y Lehrer (1997).
} 
BRANIGAN, Edward R. (1992): Narrative Comprehension and Film. Routledge. Londres y Nueva York.

BRECHER, Elinor J. (1994): Schindler's Legacy. Hodder \& Stoughton. Londres.

BRESHEETH, Haim (1997): "The Great Taboo Broken: Reflections on the Israeli Reception of Schindler's List' en LOSHITZKY, Yosefa (ed.): Spielberg's Holocaust: Critical Perspectives on Schindler's List (pp. 192-212). Indiana University Press. Bloomington e Indianápolis.

COPPOLA, Francis Ford (dir.) (1983): Rumble Fish. Hotweather Films y Zoetrope Studios. EE.UU. [Título traducido en España como La ley de la calle].

FENSCH, Thomas (1995a): "The Journalist Who Knew Oskar Schindler: An Interview with Herbert Steinhouse" en FENSCH, Thomas (ed.): Oskar Schindler and His List: The Man, the Book, the Film, the Holocaust and Its Survivors (pp. 3-19). Paul S. Eriksson. Forest Dale, Vermont.

FENSCH, Thomas (ed.) (1995b): Oskar Schindler and His List: The Man, the Book, the Film, the Holocaust and Its Survivors. Paul S. Eriksson. Forest Dale, Vermont.

GIANNETTI, Louis (1996): Understanding Movies. Prentice Hall International. Nueva York.

KENEALLY, Thomas (1993) [1982]: Schindler's List. Hodder and Stoughton. Londres. [Publicado originalmente como Schindler's Ark].

LANHAM, Fritz (1995): "Keneally's Luck" en FENSCH, Thomas (ed.): Oskar Schindler and His List: The Man, the Book, the Film, the Holocaust and Its Survivors (pp. 41- 
44). Paul S. Eriksson. Forest Dale, Vermont. [Publicado originalmente en (1995): en Periódico Houston Chronicle, 24 de abril].

LEHRER, Natasha (1997): "Between Obsession and Amnesia: Reflections on the French Reception of Schindler's List" en LOSHITZKY, Yosefa (ed.): Spielberg's Holocaust: Critical Perspectives on Schindler's List (pp. 213-225). Indiana University Press. Bloomington e Indianápolis.

PONCE, Pedro E. (1995): "Making Novels of Life's Ethical Dilemmas" en FENSCH, Thomas (ed.): Oskar Shchindler and His List: The Man, the Book, the Film, the Holocaust and Its Survivors (pp. 38-40). Paul S. Eriksson. Forest Dale, Vermont. [Publicado originalmente en (1994): en Revista The Chronicle of Higher Education, 2 de febrero].

SEGER, Linda (1993): El arte de la adaptación. Rialp. Madrid. [Traducción de Albert Méndiz. Publicado originalmente en (1992): The Art of Adaptation. Henry Cold and Company, Inc. Nueva York].

SPIELBERG, Steven (dir.) (1972): Duel. Universal TV. EE.UU. [Estrenada previamente el 13 de noviembre de 1971 en una versión más breve para la televisión. Título traducido en España como El diablo sobre ruedas].

SPIELBERG, Steven (dir.) (1982): E.T. The Extraterrestrial. Universal Pictures. EE.UU. [Título traducido en España como E.T. El extraterrestre].

SPIELBERG, Steven (dir.) (1987): Empire of the Sun. Amblin Entertainment y Warner Bros. Pictures. EE.UU. [Título traducido en España como El imperio del sol].

SPIELBERG, Steven (dir.) (1993): Schindler's List. Amblin Entertainment y Universal Pictures. Estados Unidos. [Título traducido en España como La lista de Schindler]. 
STEINHOUSE, Herbert (1995): "The Man Who Saved a Thousand Lives" en FENSCH, Thomas (ed.): Oskar Shchindler and His List: The Man, the Book, the Film, the Holocaust and Its Survivors (pp. 20-35). Paul S. Eriksson. Forest Dale. Vermont.

THOMPSON, Anne (1995): "Making History: How Steven Spielberg Brought "Schindler's List" to Life" en FENSCH, Thomas (ed.): Oskar Shchindler and His List: The Man, the Book, the Film, the Holocaust and Its Survivors (pp. 65-74). Paul S. Eriksson. Forest Dale. Vermont. [Publicado originalmente en (1994): en Revista Entertainment Weekly, 21 de enero].

WEISSBERG, Liliane (1997): "The Tale of a Good German: Reflections on the German Recepction of Schindler's List" en LOSHITZKY, Yosefa (ed.): Spielberg's Holocaust: Critical Perspectives on Schindler's List (pp. 171-192). Indiana University Press. Bloomington e Indianápolis. 